\title{
Human Body Measurement by Digital Photogrammetry System
}

\author{
Nobuo KOCHI*, Kazuo KITAMURA, Hiroto WATANABE, Takayuki NOMA, Mitsuharu YAMADA \\ Imaging and Measuring Laboratory, R\&D Center, Topcon Corporation, Tokyo, Japan
}

\begin{abstract}
We have developed an automatic system of all around 3D measurement comprised of special digital cameras and projector in a unit, which can measure human body all around with high precision. It has a calibration box which we use, prior to photographing, to determine the exterior orientation parameters of the cameras so that 3D measurement can be performed automatically as soon as the photographing unit is set up against an object. For stereo-matching we created a robust matching system and integrated it into an all-automatic system.
\end{abstract}

\section{Introduction}

We have been working on Close Range Digital Photogrammetry and on the related software (Topcon PI-3000, Image Master) for 3D measurement to process stereo-images. And we have applied it to various kinds of measurement from height altitude air plane to helicopter photogrammetry, as well as to the surface measurement. The software works stereo measurement with more than two stereoimages. Its camera can be one or many. Here are some examples of its application on land, building, automobile etc, of various sides from several hundred meters down to 10 or even less [1 3].

Where it is possible to set up more than two cameras, this system works well with a moving object like human body. Even for surface measurement, stereo-method works better than other active methods such as Laser, because with stereo-method, we can take and record pictures with high speed shutter [4].

With this back ground, this time, we applied our method specifically to the measurement of a human body, by constructing an automatic system with stereo-method as its basic principle and developing a software of automatic measurement.

So we constructed a photographing unit with a special small projector and two digital cameras. And we used four of them together to set up around a human body.

For the preparatory operation, however, we created a calibration box, covered with color-coded targets, the automatic detection of which enable us to make the exterior orientation parameters of each units automatically. The rest of the 3D measurement operation was also handled all automatically by way of forward intersection principle.

As to the stereo-matching process (automatic surface measurement), we have developed a system, which enable us to obtain highly accurate 3D measurement by LSM (Least Square Matching) [5] with the 3D data obtained by OCM (Orientation Code Matching) [6] as the initial value. And as to LSM, while it does well with geometrical deviation, it takes too much time [7]. To avoid that, it needs the initial value of fairly high precision. On the other hand, OCM is robust vis-à-vis the deformation, occlusion as well as brightness change and as such, it is much better than any other prevailing methods for stereo-matching [8].

In this study, therefore, we are presenting the report on our system of human body measurement and its processing method of stereo-matching..

\section{Photo-Measuring System}

Our system consists of photographing units, each of which is composed of two special digital cameras and a projector (Fig. 1 Left). We have also created a calibration box for preparatory process (Fig. 1 Middle). For both preparatory process and 3D measurement processing we use PC.

For preparatory process, we take the picture of a calibration box to obtain the exterior orientation parameters of the cameras. Therefore, we do not have to paste the target labels on the object itself. Not only that, we can now make subsequent process of 3D measurement all automatic.

*n.kochi@topcon.co.jp 

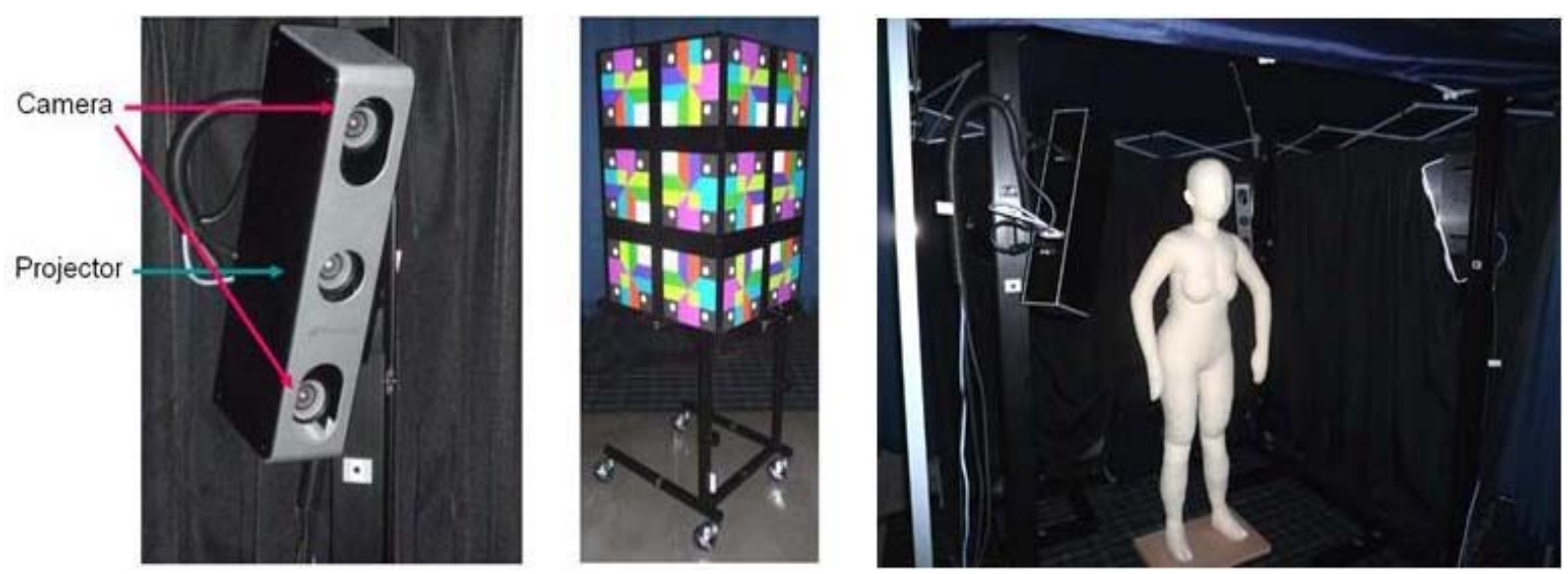

Fig. 1. Human Body Measurement System: Photographing Unit (Left), Calibration Box (Middle) and Whole System (Right)

And here all the cameras are synchronized and we can obtain a number of pictures at the same instant. And in order to make stereo-matching process on the surface of 3D measurement precise, we project patterns on the object, take its pictures and process analytically the obtained data by PC. If we need texture (object image), we take the picture without projecting patterns and then add the texture over the measurement result.

For a human body, we use 4 photographing units around the body. Fig. 1 Right shows this human body measurement system.

\section{Flow of Measurement}

The flow of measurement process is shown in Fig. 2. 1) $\sim 3$ ) is the preparatory process, 4 ) $\sim 6$ ) is the 3D measurement process.

1) First, in the preparatory process, we take the picture of Calibration Box pasted with color-coded targets with each unit. Since pattern projection for an object is not necessary, the Box is lit just once by the projectors without patterns, which otherwise would be projected on an object and the result is fed into PC. The 3D measurement of the Box with color coded targets should be made with high precision and equalize the code numbers with their coordinates.

2) Next, the color-coded targets pasted on the Box are automatically detected. Using our automatic Color-Code Detection Software which we have developed, we detect automatically the position and code number of the color codes [9] and equalize them with the correspondent 3D coordinates of the Calibration Box, which have been previously measured and determined.

3) We calculate the exterior orientation parameters of each Photographing Unit by the corresponding code number and its 3D position. After the preparatory process of 1 ) $\sim 3$ ), automatic 3D measurement becomes successively possible for any number of human body.

4) We take the picture of a human body by Photographing Units. For 3D measurement we project random dot patterns on the body. If we wish to put texture on the result of $3 \mathrm{D}$ measurement, we take another picture without patterns. Since the cameras can take synchronously instantly, the alternation of the patterns is also possible instantly. So we can take the images with patterns and without patterns almost at the same time. The Fig. 3 shows the two pictures: one with and another without patterns.

5) We make 3D measurement by Stereo-Matching. Here we can match enormous amount of points in the order of thousand and ten thousands, and this, all automatically. We do this on the Stereo-Image of each photographing unit. As to the Stereo-Matching Method, we will explain in the next chapter.

6) We output 3D data. 


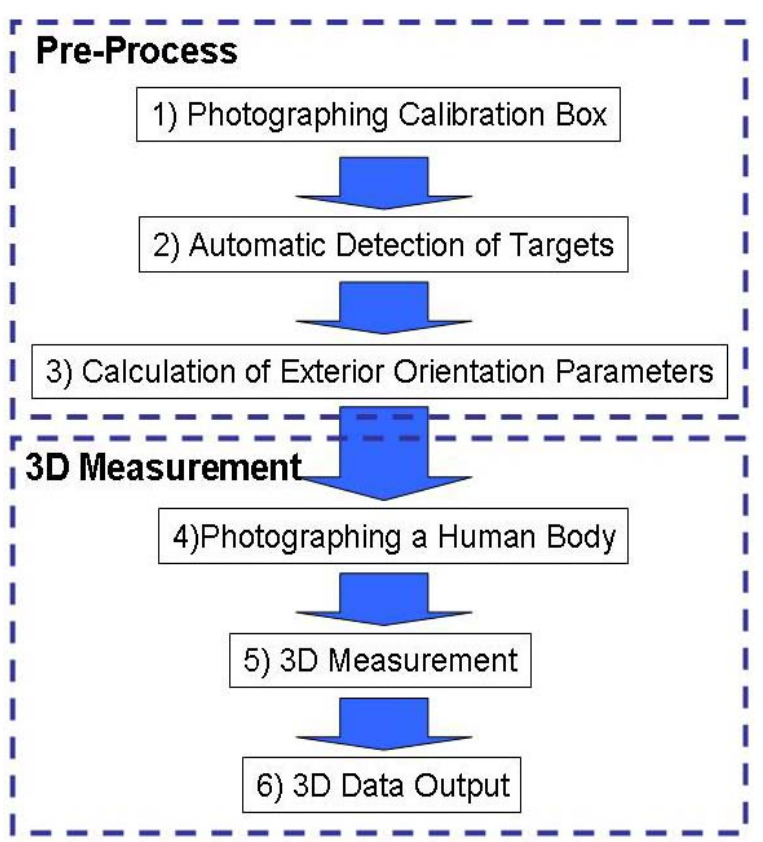

Fig. 2. Flow of Measurement

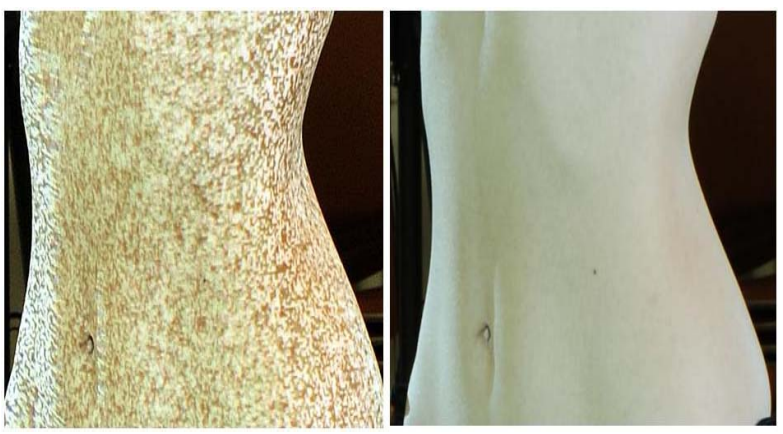

Fig. 3. With Patterns (Left), Without Patterns (Right)

\section{Stereo-Matching}

\subsection{Basic Principle}

We used to apply LSM method for stereo-matching. It is robust for geometrical deformation, but it needs the initial value of fairly high precision. The reason is that as LSM makes matching while changing the search area, unless it has the initial value of sufficient accuracy, not only the frequent change of forms demands longer time for processing, but also it is liable to error (mis-matching) because it makes futile deformation.

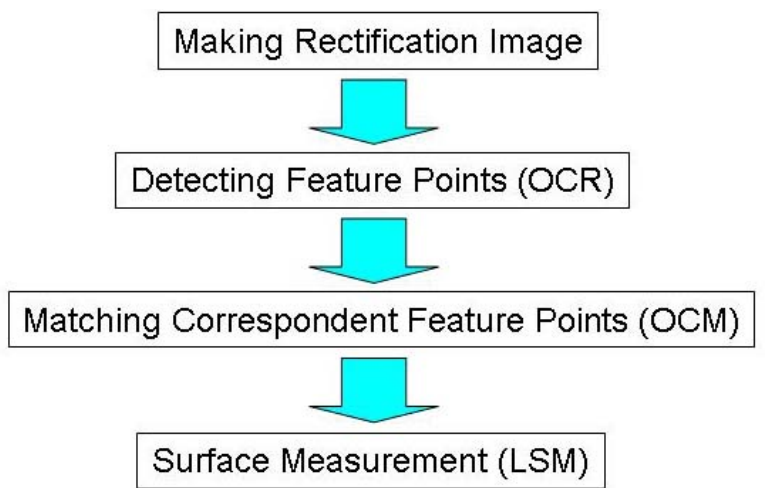

Fig.4. Process Flow

In the past, we used to input the initial value by manual or semi-automatic operation [10], which was complicated. But this time we created an automatic stereo-matching system to detect the feature points by means of OCR (Orientation Code Richness) and make them the initial value for LSM.

The Fig. 4 is the flow of the process. First we make rectification image and detect the feature points of the left rectification image by OCR. And then we make templates around the detected points and search the corresponding points on the right rectification image by template-matching of OCM. And finally, with the corresponding feature points on the left and right image as the initial value, we make the surface measurement by LSM (which means another precise stereo-matching). 


\subsection{Orientation Code (OC)}

We use the feature quantity, which is called Orientation Code $(\mathrm{OC})$, both for feature point detection and for matching. We define the quantified value of orientation in the adjacent pixels as OC.

Suppose if we determine the intensity at the pixel coordinates $(x, y)$ of an object as $I(x, y)$ and if we determine the intensity gradient on the horizontal axis as $\Delta \mathrm{I}_{\mathrm{x}}=\partial \mathrm{l} / \partial_{\mathrm{x}}$, and the intensity gradient on the vertical axis as $\Delta \mathrm{l}_{\mathrm{y}}=\partial \mathrm{l} / \partial_{\mathrm{y}}$, then we can obtain the orientation angle of the interest pixel as $\theta(\mathrm{x}, \mathrm{y})=\tan ^{-}$ ${ }^{1}\left(\Delta \mathrm{l}_{\mathrm{y}} / \Delta \mathrm{I}_{\mathrm{x}}\right)$. In this study we used Sobel operator [11] to calculate the gradient value. The Orientation Code is the quantized value of the orientation angle $\theta$ within the proper quantize width $\Delta \theta=2 \pi / \mathrm{N}$. Its equation is as follows.

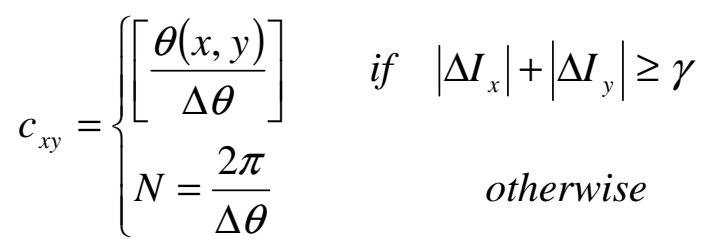

We quantize the periphery of a circle by $\mathrm{N}$ parts, the $\mathrm{OC}$ is shown as $0 \sim \mathrm{N}-1$. " $\mathrm{Y}$ " is the threshold value set up to obtain $\mathrm{OC}$ in stable manner and in case of low contrast we put $\mathrm{N}$ as the unreliable code. Therefore, in our study we divided the periphery by 16 directions $(\mathrm{N}=16$ : Fig. 5). Fig. 7 is the OC image of the Fig. $6(\mathrm{~N}=16, \mathrm{y}=20)$.
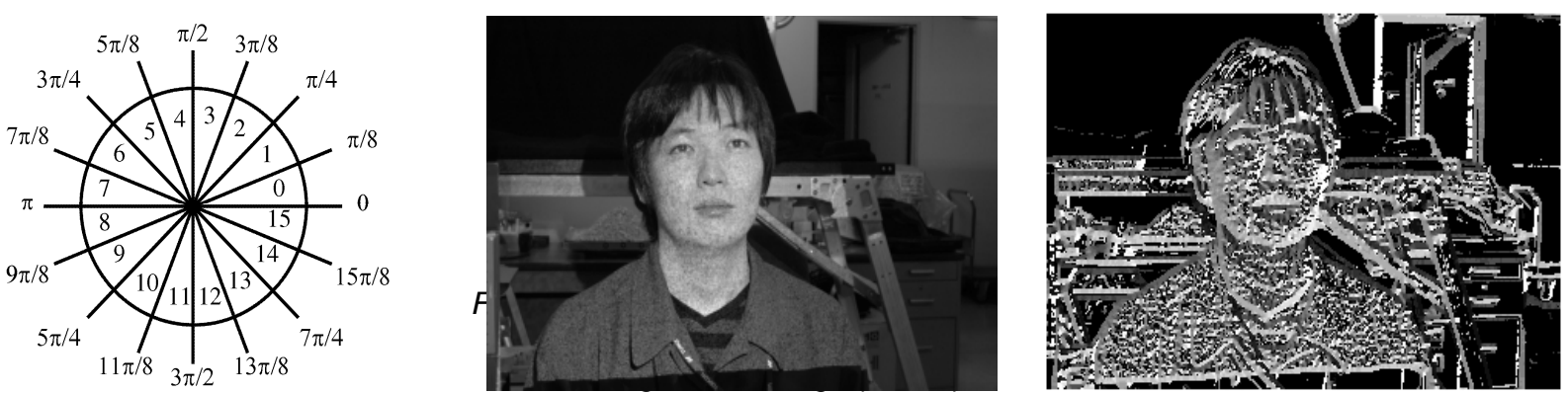

\subsection{Detection of the Feature Points}

For the detection of the feature points we used feature quantity called OCR (Orientation Code Richness) [12]. As this method detects specifically the area where there are many elements of diverse direction, it makes it easy to select the area where there is a rich texture. Besides, as this method calculates with the OC, it gives stability to the matching of OC.

We calculate the relative frequency $P_{x y}(i)=h_{x y}(i) / M^{2}-h_{x y}(N)(i=0,1,,,,,, N-1)$ with the frequency of the apparition of each $O C$ as $h_{x y}(i)(i=0,1,,,,,, N-1)$ in a limited local area of the pixel size $M$ by $M$ region centered at the interest pixel $(x, y)$. At this time, since the value of the $h_{x y}(N)$ is the unreliable code for the low contrast, we leave it out from the relative frequency. As a result, the entropy of OC, $0 \sim \mathrm{N}-1$, is shown by the following equation.

$$
E_{x y}=\sum_{i=0}^{N-1} P_{x y}(i) \log _{2} P_{x y}(i)
$$

The maximum value of entropy $E_{\max }$ is $E_{\max }=\log _{2} N$, when each $\mathrm{OC}$ goes with uniform distribution $P_{x y}(i)=1 / N$. When we calculate the Richness, therefore, we set up the threshold value $\alpha_{e}\left(0<\alpha_{e}<1\right)$ and consider the area where the entropy is above $\alpha_{e} E_{\max }$ as the feature area. Thus the Richness $R_{x y}$ is shown in the following equation.

$$
R_{x y}= \begin{cases}\frac{E_{x y}-\alpha_{e} E_{\max }}{E_{\max }-\alpha_{e} E_{\max }} & \text { if } E_{x y} \geq \alpha_{e} E_{\max } \\ 0 & \text { otherwise }\end{cases}
$$

We made OCR image by calculating the Richness of the entire picture and covered it with grids of the predetermined size, and detected the highest Richness of each grid as the feature points (Fig. 8). 
In this study we first detect the feature points on the left rectification image and then detect the corresponding points on right rectification image by using the specific method to compare Orientation Code, as explained in the next chapter.

The Fig. 8 shows the OCR image of Fig. 6 . We picked out the maximum value in each grid as the feature point. The Fig. 9 shows the detected feature points with a mark.

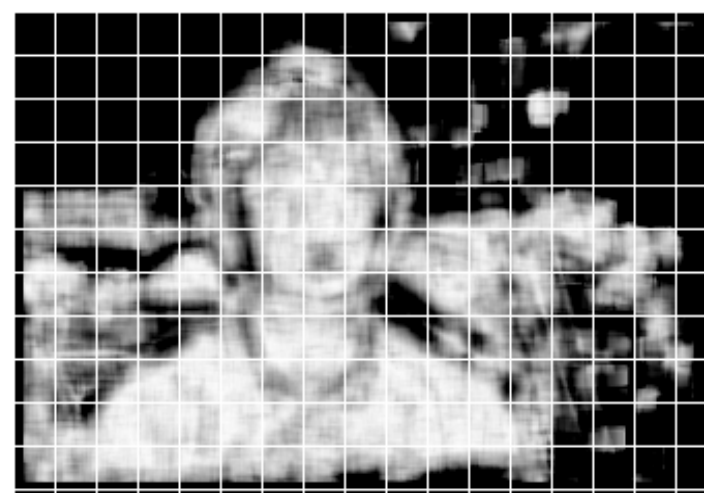

Fig. 8. OCR Image

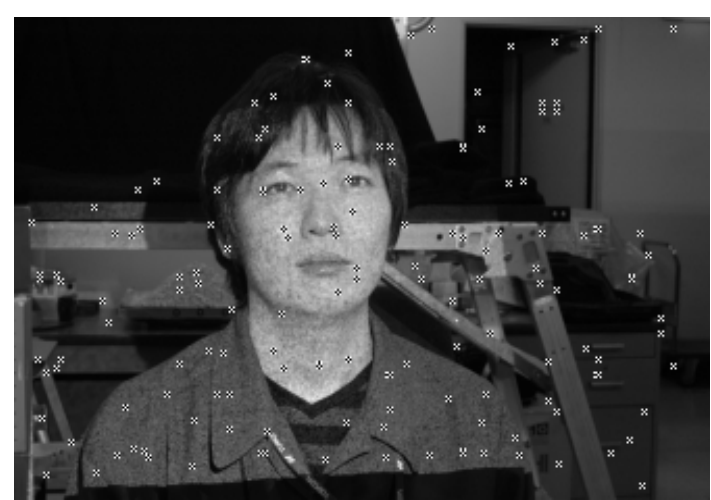

Fig. 9. Result of Feature Points Detection

\subsection{Feature Points Matching (Orientation Code Matching)}

To match the corresponding feature points we applied the matching method, which uses $O C$ as explained in 4.2 .

Suppose if we give the name "O" to the original image, to which the other image "R" is referred, both having the same size $S_{x} \times S_{y}$, and if we make the OC Image from both of them as $\mathrm{CO}$, and $\mathrm{cR}$, by using equation (1), the following will be the relevant equation, in which the mean of absolute residuals " $D$ " works as the verification value of the absolute difference criterion values "d"

$$
\begin{aligned}
& D=\frac{1}{S_{x} \times S_{y}} \sum_{S_{x} \times S_{y}} d\left(c_{R}, c_{O}\right) \\
& d(a, b)= \begin{cases}\min \{|a-b|, N-|a-b|\} & \text { if } a \neq N, b \neq N \\
N / 4 & \text { otherwise }\end{cases}
\end{aligned}
$$

In this study we first made detection of the feature points, as we explained in the previous chapter, on the Left image. Then we made templates around the each feature point and searched the corresponding points on the Right image, using OCM.

\section{Surface Measurement}

The Fig. 10 and 11 are the all-around measurement result of the models taken from 4 different angles, using 4 photographing units. The Fig. 10 shows 3D models. And over its result we added texture mapping, which created the image as in the Fig. 11 .

To assess the accuracy of our system, we took picture of the object with Standard Scale Bar. The deviation was less than $3 \mathrm{~mm}$. As to the measuring time, from the photographing to the processing time is 90 seconds.

The result was fully satisfactory. 

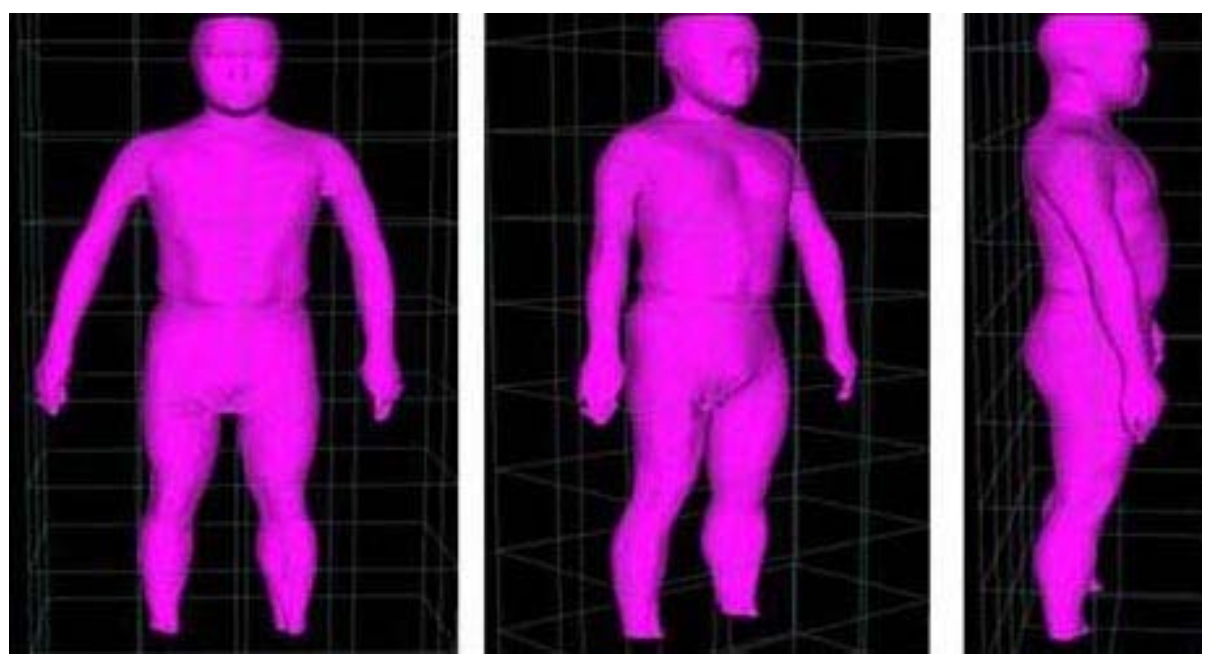

Fig. 10. 3D Model
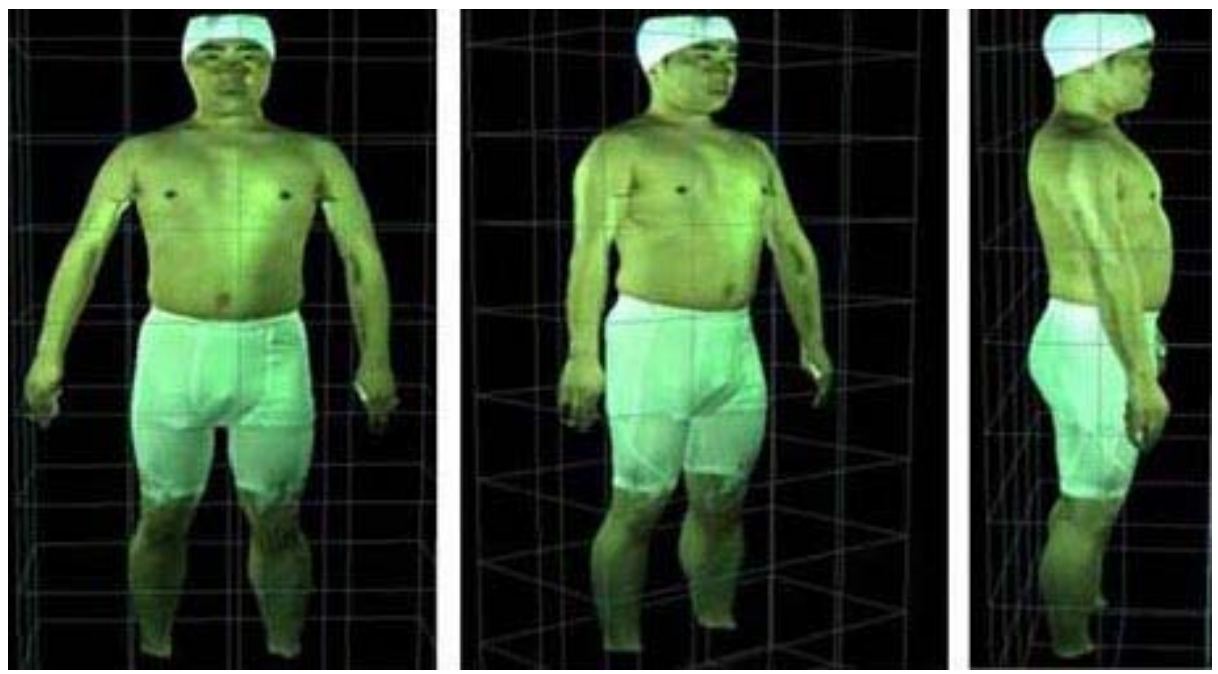

Fig 11. Texture Mapping Model

\section{Conclusion}

We created a system to make automatically the all-around 3D measurement on a human body, using special digital cameras and projectors. For this system we first made a calibration Box of the size matching that of the object to prepare the parameters of the photographing units. And with thus prepared units set-up around the object, we can make automatically the 3D measurement. Besides, by synchronizing cameras we succeeded to make it possible to create 3D measurement of a moving object like a human body.

For Stereo-Matching, we created a new robust system fully automatic, combining OCM and LSM. In order to obtain automatically the initial values we invented a system, in which we can detect and correlate the feature points in and between OCR and OCM with much higher accuracy. And using this initial values, we perform the stereo matching by LSM and thus succeeded not only make the surface measurement highly accurate but also to minimize the measuring time.

In future, therefore, we should like to study and develop its various useful applications. 


\section{References}

[1] Kochi, N., Watanabe, H., Ito, T., Otani, H. and Yamada, M., (2004): "3Dimensional Measurement Modeling System with Digital Camera on PC and its Application Examples", International (SPIE) Conference on Advanced Optical Diagnostics in Fluids, Solids and Combustion, December 4-6,Tokyo, Japan

[2] Kochi, N., Yamada, M., Watanabe, H., Aoki,H., (2005):"3D-Measuring-Modeling-System based on Digital Camera and PC to be applied to the wide area of Industrial Measurement"、The International Society for Optical Engineering(SPIE) Conference on Optical Diagnostics, August,Sandiego,USA

[3] Kochi, N., Photogrammetry, Handbook of Optical Metrology : Principles and Applications, Yoshizawa,T.'(Ed.), Taylor and Francis, NewYork, 2009, Chapter 22.

[4] D’Apuzzo, N., and Mitchell, H., Medical,(2008): "Applications, Advance in Photogrammetry, Remote Sensing and Spatial Information Science",ISPRS Congress Book., Li, Z., Chen, J., and Baltsavias, E., CRC Press, London, 2008, chap.31

[5] Grun, A., (1985):"Adaptive Least-squares correlation: a powerful image matching technique." South Africa Journal of Photogrammetry, Remote Sensing and Cartography, 14(3), 175-187

[6] Ullah, F., Kaneko, S., and Igarashi, S.,(2001): "Orientation code matching for robust object search", IEICE Trans. of Inf. \& Sys, E84-D(8), pp.999-1006.

[7] Shenk, T., (1999): "Digital Photogrammetry Vol1", Terra Science, pp237-263

[8] Domae, Y., Takauji, H., Stier, H., Kaneko, S., Tanaka, T., (2006):"Extraction and Tracking Of Orientation Coded Features Being Robust Against Illumination Changes", Proceedings of the ISPRS Commission V Symposium, Vol. XXXVI, Part5, Dresden, Germany, September

[9] Moriyama, T., Kochi, N., Yamada, M., Fukaya, N., (2008): "Automatic Target-Identification with the Color-Coded-Targets", The International Archives of Photogrammetry and Remote Sensing, Beijing, XXI Congress, WG V/I, pp.39-44

[10] Kochi, N., Ito, T., Noma, H., Otani, Nishimura, S., Ito, J. (2003): "PC-Based 3D Image Measuring Station with Digital Camera an Example of its Actual Application on a Historical Ruin" International Archives of Photogrammetry and Remote Sensing, Vol.XXXIV-5/W12, Ancona, p.117-120

[11] Sobel, I. (1978): Neighbourhood coding of binary images for fast contour following and general array binary processing", Computer Graphics and Image Processing, vol.8, pp.127-135.

[12] Takauji, H., Kaneko, S. and Tanaka, T. (2005): "Robust tagging in strange circumstance", The Transactions of the Institute of Electrical Engineers of Japan (C), vol.125, pp.926, (in japanese) 